\title{
A novel method for pair-matching using three-dimensional digital models of bone: mesh-to-mesh value comparison
}

\author{
Mara A. Karell ${ }^{1,2}$ • Helen K. Langstaff ${ }^{1,2}$ • Demetrios J. Halazonetis ${ }^{3}$. \\ Caterina Minghetti ${ }^{4}$. Mélanie Frelat ${ }^{4,5,6}$ - Elena F. Kranioti ${ }^{1,2}$
}

Received: 9 July 2015 / Accepted: 10 February 2016 / Published online: 10 March 2016

(C) The Author(s) 2016. This article is published with open access at Springerlink.com

\begin{abstract}
The commingling of human remains often hinders forensic/physical anthropologists during the identification process, as there are limited methods to accurately sort these remains. This study investigates a new method for pair-matching, a common individualization technique, which uses digital three-dimensional models of bone: mesh-to-mesh value comparison (MVC). The MVC method digitally compares the entire three-dimensional geometry of two bones at once to produce a single value to indicate their similarity. Two different versions of this method, one manual and the other automated, were created and then tested for how well they accurately pairmatched humeri. Each version was assessed using sensitivity and specificity. The manual mesh-to-mesh value comparison method was $100 \%$ sensitive and $100 \%$ specific. The automated mesh-to-mesh value comparison method was $95 \%$ sensitive
\end{abstract}

Electronic supplementary material The online version of this article (doi:10.1007/s00414-016-1334-3) contains supplementary material, which is available to authorized users.

Mara A. Karell

marakarell@gmail.com; m.a.karell@sms.ed.ac.uk

1 Edinburgh Unit of Forensic Anthropology, Edinburgh, UK

2 School of History, Classics and Archaeology, University of Edinburgh, Edinburgh, UK

3 Department of Orthodontics, School of Dentistry, National and Kapodistrian University of Athens, Athens, Greece

4 Department of Cultural Heritage, University of Bologna, Ravenna, Italy

5 UMR 7268 ADES, Aix-Marseille Université/EFS/CNRS, Marseille, France

6 Evolutionary Studies Institute, University of the Witwatersrand, Johannesburg, South Africa and $60 \%$ specific. Our results indicate that the mesh-to-mesh value comparison method overall is a powerful new tool for accurately pair-matching commingled skeletal elements, although the automated version still needs improvement.

Keywords Forensic anthropology · Pair-matching · Commingled remains $\cdot 3 \mathrm{D}$ geometry $\cdot 3 \mathrm{D}$ modeling . Mesh-to-mesh value comparison

\section{Introduction}

In forensic anthropology, pair-matching skeletal elements is a common individualization technique for situations involving commingled remains, such as mass graves, or mass tragedies due to natural disasters. Currently, techniques range from visual pair-matching, which is a subjective technique based on the similarities in morphology of left and right paired elements [1], to more objective methods such as osteometric comparison, which uses statistical models and quantitative approaches to compare the size and morphology between elements, paired or not [1-3]. More recently, a geometric morphometric approach has also been applied to the task [4].

All of these methods' accuracy rates, however, vary considerably. In the only two studies conducted on the method, visual pair-matching ranges from being roughly 75 to $90 \%$ accurate, depending of the type of skeletal element being matched $[4,5]$. A geometric morphometric approach seems to be more reliable, at $100 \%$ accurate, but this is only the case if all true pair matches are actually present in the sample population [4]. Otherwise, this method will lead to erroneous results [4]. Furthermore, to our knowledge, there has only been one study conducted on the topic, focused only on metacarpals [4]. Osteometric comparison methods have in some ways side-stepped the problem of matching paired elements by 
focusing instead on using a null hypothesis which is based on the principle of exclusion [6]. Thus, the accuracy of excluding that two skeletal elements match is predicted using either 90th and 95 th percentiles or a $90 \%$ prediction interval, but again appears to be variable in terms of reliability when applied to different skeletal populations [6-8].

The fact that these methods' accuracy rates vary considerably is not surprising; the accuracy of visual pair-matching often depends on the experience of the osteologist, while both visual pair-matching and osteometric comparison methods acknowledge that anatomical similarity between individuals often complicates individualization. Furthermore, despite the knowledge that paired skeletal elements from the same individual are often quite asymmetrical, currently only the geometric morphometric approach acknowledges the effects of bilateral asymmetry with regard to pair-matching $[4,9,10]$. Additionally, as the geometric morphometric approach relies on the consistent landmarking of variable anatomical features, there is always the complication of ensuring that each practitioner can reliably identify and place the required anatomical landmarks, without introducing error [11].

Beyond this variability in the accuracy of pair-matching, it is also important to note the recent push in forensic/physical anthropology to make all individualization methods more objective and reliable, with known error rates [12]. This push paired with the increased use of technology, such as CT and 3D surface scanners, throughout the field of forensic anthropology [13-15] suggests that new technologies could be used to help improve current individualization techniques such as pair-matching, creating more objective and reliable methods, with known error rates.

In this vein, we propose a new method of pair-matching, mesh-to-mesh value comparison (MVC), which directly compares the entire digital three-dimensional morphology of two bones without the need for biological landmarking. This method utilizes a "mesh-to-mesh value," a single value which quantifies the difference of two meshes (models) in millimeters, though it is not necessarily a simple average of the distances between the two models. Still, the lower a mesh-to-mesh value, the more similar two models, or meshes are. The algorithms used to determine a mesh-to-mesh value are based on Iterative Closest Point (ICP) comparison algorithms [16]. For the initial manual MVC method, using LMI Technologies Flexscan 3D software, other, unknown, proprietary algorithms are also involved in mesh-to-mesh value creation. To account for this, we designed an automated MVC method using an add-on for the software program Viewbox 4 (dHAL Software, Kifissia, Greece), in which the algorithms used to compare mesh similarity could be controlled and the process sped up.

In addition to testing the MVC method, sensitivity and specificity rates were assessed as an alternative analysis tool for accuracy. Though this method of analysis has previously only been used in forensic science for the comparison of diagnostic tests such as the presence of drugs [17] and human saliva [18], it was chosen for this study because it offers more information than simply counting accurate pair matches alone. Instead, it demonstrates the accuracy of a method by giving the precise rates of a method choosing, for example, false pair matches verses true pair matches or true negatives. In this way, pair-matching methods, regardless of the population size, number of pairs, and number of single bones, can be easily cross-compared and understood. Additionally, though current methods generally report type I errors, sensitivity and specificity rates express both type I and type II errors, the latter of which is an underused statistic in biological anthropology [19]. Sensitivity and specificity rates also provide greater information about how a method may have misidentified some pairs, elucidating if it was mismatching bones or if it simply did not find a match for a bone at all.

The aim of this study was to determine if digital threedimensional models of bones could be used to pair-match skeletal elements with a high degree of statistical accuracy, as measured by sensitivity and specificity. This was tested utilizing two different versions of the MVC method, one manual and the other automated. Paired humeri were chosen as a test population on the reasoning that if a method can accurately sort highly asymmetrical unmatched humeri into their proper pairs, it can aid in sorting all paired bones. In this manner, the study acknowledged bilateral asymmetry and its possible effect on pair-matching.

\section{Materials and methods}

\section{Materials}

A total of 45 well-preserved humeri from three different populations (G1, G2, and G3) were used for this study, to insure coverage of multiple time periods and geographical locations. Thirty-one humeri were scanned via computed tomography (CT) and 14 humeri were three-dimensional surface scanned. The main difference between the CT and 3D surface scan data was that the $3 \mathrm{D}$ surface data does not include the internal structures of the objects which were scanned [20].

Ten known pairs of humeri (G1) originated from the Ballumbie and St. Andrews medieval Scottish collections (fifteenth to seventeenth century) held by the University of Edinburgh [21, 22]. Eleven humeri (G2), including four pairs and three individual humeri, originated from the archaeological Ibizan cathedral collection (thirteenth to early nineteenth century) in Spain, held by the Ibizan city hall [23]. Seven known pairs of humeri (G3) originated from the Frassetto Collection (Collezione Frassetto) in Italy, a modern collection held by the Anthropology Museum at the University of Bologna. All of these individuals originated from Sassai (Sardinia), died in the first decade of the twentieth century, 
and were donated to the collection. For a full list of specimens, see Table 1.

\section{Scanning protocols}

CT scans of G1 were taken at the Clinical Research Imaging Centre, University of Edinburgh, using a Toshiba Aquilion ONE 320 Detector Row Computed Tomography system, a multidetector CT scanning system. Data were collected using a slice thickness of $0.5 \mathrm{~mm}$ and a matrix of $512 \times 512$ pixels. CT scans of G2 were taken at the Can Misses Hospital, Ibiza, using a GE Medical System HiSpeed NX/I Computed Tomography Scanner. Data were collected using a slice thickness of $1.5 \mathrm{~mm}$ and a matrix of $512 \times 512$ pixels. All data were saved as a Digital Imaging and Communications in Medicine (DICOM) format.

Three-dimensional surface scans of the humeri in G3 were made using a two camera system, each 1.9 megapixels, with a ScanProbe Standard structured light scanner at the University of Bologna. After the initial data acquisition and aligning phases, the point cloud models were input into XOR2 software to generate the final models and saved as stereolithography (.stl) files.

All scan data were randomized before the pair-matching process was tested to minimize bias.

\section{Segmentation}

After randomization, the 31 humeri of G1 and G2 were segmented using AMIRA 5.3.3 to create three-dimensional models using a slightly modified version of Spoor et al.'s Half Maximum Height Value (Online Resource 1) [24]. The 14 humeri from G3 were already three-dimensional surface models and therefore did not need to be segmented. All of the three-dimensional models were then converted from their stereolithography [.stl] format to wavefront [.obj] files.

\section{Mesh-to-mesh value comparison method — manual}

The manual MVC method utilized LMI Technologies' Flexscan 3D to compare all the humeri. The protocol for comparison is as follows:

All right humeri were mirror-imaged using the free software NetFabb basic. All mirror-imaged humeri were then loaded into the Flexscan3D software. One left humerus at a time was then also loaded into the software for comparison against all of the mirrored humeri. All 22 left humeri were subsequently compared to all 23 mirrored-right humeri. To compare any two humeri, both scans were roughly lined up on top of each other using the mouse. Then, the "fine alignment" feature was used in order to obtain a mesh-to-mesh value, which was recorded for comparison (see Fig. 1 for an example).
The mesh-to-mesh values were used as a proxy for pairmatching, where the lowest agreed-upon value indicated the best match. The side of the humeri was initially used to narrow down these values, as a left humerus could not be pairmatched with another left humerus. For the actual test of pair-matching, the three lowest mesh-to-mesh values of each humerus were cross-compared and values were only considered as true matches if both the left and right sides agreed. This was done in order to avoid confusion, for example, if Left Humerus $A$ indicated that it matched best with Right Humerus B, but Right Humerus B indicated that it matched best with Left Humerus C. The standard deviations of meshto-mesh values from true pair-matches were calculated to inform a possible cutoff threshold for positive pair matches. In total, comparing all of the humeri to obtain mesh-to-mesh values and recording said values took approximately 45 user-active hours.

\section{Mesh-to-mesh value comparison method — automated}

The automated MVC method utilized Viewbox 4 for comparison. The following settings were used, comparing all meshes to each other from a single folder. The estimated overlap for the scans was $100 \%$, while the initial position for rough alignment was set at 20 . The rough alignment used the nearest neighbor search "Approximate (fast)" with a point sampling of $1 \%$. It matched point to point, with one hundred iterations. The fine alignment used the nearest neighbor search "Exact with normal compatibility" with a point sampling of $100 \%$. It matched point to plane, with one hundred iterations. The program then automatically generated an Excel spreadsheet of all of the mesh-to-mesh values for analysis. It should be noted that as this program cannot yet handle comparing $3 \mathrm{D}$ surface scans to full CT scan data, the $31 \mathrm{CT}$ scan models were internally hollowed before comparison.

Again, the mesh-to-mesh values were used as a proxy for pair-matching, where the lowest agreed-upon value indicated the best match. The side of the humeri was initially used to narrow down these values. For the actual test of pair-matching, the three lowest mesh-to-mesh values of each humerus were cross-compared, and values were only considered as true matches if both the left and right sides agreed. The standard deviations of mesh-to-mesh values from true pair-matches were calculated to inform a possible cutoff threshold for positive pair matches. The process took approximately five minutes to set up and then approximately 45 hours to run. Only the first five minutes of set up required any activity from the user.

\section{Comparison of the methods}

To compare the efficacy of the two MVC methods, specificity and sensitivity were calculated using Microsoft Excel 2007 [25]. For this study, the gold standard method for comparison 
Table 1 List of specimens including sex, age, and completeness

\begin{tabular}{|c|c|c|c|c|c|}
\hline ID number & Randomized number & Collection & Age & Sex & Completeness ${ }^{\mathrm{a}}$ \\
\hline StA-17R & 8 & Scotland & Adult & - & 1 \\
\hline StA-17 L & 4 & Scotland & Adult & - & 1 \\
\hline StA-26R & 1 & Scotland & Adult & - & 1 \\
\hline StA-26 L & 14 & Scotland & Adult & - & 1 \\
\hline StA-35R & 17 & Scotland & Adult & - & 1 \\
\hline StA-35 L & 19 & Scotland & Adult & - & 1 \\
\hline StA-36R & 25 & Scotland & Adult & $\mathrm{F}$ & 1 \\
\hline StA-36 L & 30 & Scotland & Adult & $\mathrm{F}$ & 1 \\
\hline StA-47R & 34 & Scotland & Adult & - & 1 \\
\hline StA-47 L & 38 & Scotland & Adult & - & 1 \\
\hline StA-61R & 50 & Scotland & Adult & $\mathrm{F}$ & 1 \\
\hline StA-61 L & 54 & Scotland & Adult & $\mathrm{F}$ & 1 \\
\hline StA-75R & 63 & Scotland & Adult & - & 1 \\
\hline StA-75 L & 3 & Scotland & Adult & - & 1 \\
\hline Bal-501R & 7 & Scotland & Adult & - & 2 \\
\hline Bal-501 L & 12 & Scotland & Adult & - & 2 \\
\hline Bal-599R & 16 & Scotland & Adult & - & 2 \\
\hline Bal-599 L & 22 & Scotland & Adult & - & 2 \\
\hline Bal-623R & 43 & Scotland & Adult & - & 1 \\
\hline Bal-623 L & 51 & Scotland & Adult & - & 1 \\
\hline Td-43R & 13 & Italy & Adult & M & 1 \\
\hline Td-43 L & 18 & Italy & Adult & M & 1 \\
\hline Td-44R & 23 & Italy & Adult & M & 1 \\
\hline Td-44 L & 27 & Italy & Adult & M & 1 \\
\hline Td-45R & 31 & Italy & Adult & M & 1 \\
\hline Td-45 L & 36 & Italy & Adult & M & 1 \\
\hline Td-46R & 39 & Italy & Adult & M & 1 \\
\hline Td-46 L & 44 & Italy & Adult & M & 1 \\
\hline Td-104R & 57 & Italy & Adult & M & 1 \\
\hline Td-104 L & 6 & Italy & Adult & M & 1 \\
\hline Td-135R & 11 & Italy & Adult & M & 1 \\
\hline Td-135 L & 20 & Italy & Adult & M & 1 \\
\hline Td-271R & 28 & Italy & Adult & M & 1 \\
\hline Td-271 L & 33 & Italy & Adult & M & 1 \\
\hline Cath-3/4R & 46 & Ibiza & Adult & - & 1 \\
\hline Cath-3/4 L & 49 & Ibiza & Adult & - & 1 \\
\hline Cath-295R & 60 & Ibiza & Adult & - & 1 \\
\hline Cath-368/369R & 2 & Ibiza & Adult & - & 1 \\
\hline Cath-368/369 L & 5 & Ibiza & Adult & - & 1 \\
\hline Cath-5 /UE 2115R & 10 & Ibiza & Adult & - & 2 \\
\hline Cath-148/149R & 15 & Ibiza & Adult & - & 1 \\
\hline Cath-148/149 L & 21 & Ibiza & Adult & - & 1 \\
\hline Cath-7/UE 2115 L & 24 & Ibiza & Adult & - & 1 \\
\hline Cath-1/2R & 29 & Ibiza & Adult & - & 1 \\
\hline Cath-1/2 L & 32 & Ibiza & Adult & - & 1 \\
\hline
\end{tabular}

${ }^{\text {a }}$ Completeness: $1=100-75 \%, 2=74.9-50 \%, 3=49.9-25 \%, 4=24.9-0 \%$ was the known humeri pairs. In other words, the main question for assessment was: is this pair of humeri a correct match?
Three previous, high quality studies on visual pairmatching, osteometric comparison, and geometric 


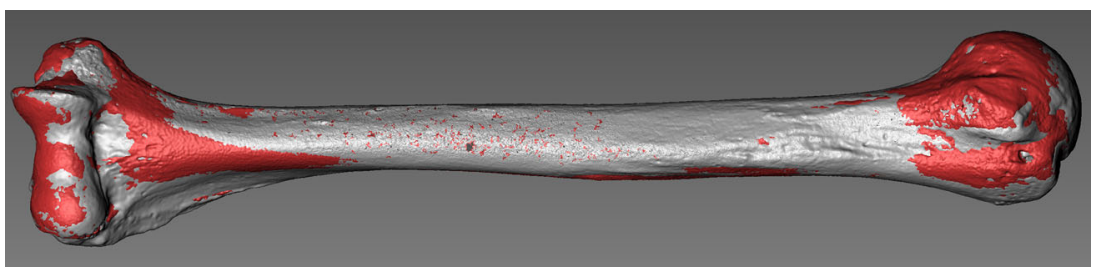

Fig. 1 Fine alignment feature. The "fine alignment" feature is used to align and compare the left and right (mirror-imaged) humeri to produce a mesh-to-mesh value. Humerus 20 (Td-135L) is pictured in red, while humerus 11 (Td-135R) is pictured in gray. The dappling of the red and

morphometric pair-matching were also analyzed using sensitivity and specificity in order to directly compare the results of the two MVC methods.

\section{Results}

For the manual version of MVC, the sensitivity and specificity were $100 \%$ and $100 \%$, respectively (Table 2). Additionally, the type of scan data did not seem to affect the accuracy of the manual MVC version. For the automated version of MVC, the sensitivity and specificity were $95 \%$ and $60 \%$, respectively (Table 3). This was because, although the automated version correctly identified all of the true negatives in the sample, it mistakenly paired Td-46L (44) with StA-75R (63) and indicated that StA-75L (3) and Td-46R (39) did not have pairmatches present; in other words, that StA-75 L (3) and Td46R (39) were negatives.

The mesh-to-mesh values of both versions were analyzed to see if a single threshold value could be used to match pairs instead of the entire Excel sheet matrix. For the manual MVC method, the average mesh-to-mesh value of all of the true matches was $0.638 \mathrm{~mm}$ and the standard deviation was $0.176 \mathrm{~mm}$. True match values ranged from 0.402 to $1.225 \mathrm{~mm}$. Two standard deviations from the mean value of $0.638 \mathrm{~mm}$ accurately captured all but one of the true match mesh-to-mesh values present. However, attempting to use this value of $1.035 \mathrm{~mm}$ as a cutoff for determining pair-matches did not work, as it included an additional 16 values which were not true matches. Similarly, for the automated MVC method, the average mesh-to-mesh value of all the true matches was $1.07 \mathrm{~mm}$ and the standard deviation was $0.310 \mathrm{~mm}$. True match values ranged from 0.524 to

Table 2 Sensitivity and specificity results for the manual mesh-to-mesh value comparison method

gray on the midshaft visually indicates a good match of the two scans, in addition to the obvious size and morphological similarities, while the mesh-to-mesh value confirms it. Image Credit: Mara Karell (color figure online)

$1.84 \mathrm{~mm}$. Two standard deviations captured all but one of the true match mesh-to-mesh values. However, attempting to use this value of $1.68 \mathrm{~mm}$ as a cutoff for determining pairmatches did not work, as it included 51 additional values which were not true matches. This suggests that utilizing the selection method of a matrix, where both the right and left sides have to agree on the lowest mesh-to-mesh value match, is a better selection method than a single threshold value, at least for humeri. For an example of a mesh-to-mesh Excel matrix and the associated calculations, see Online Resource 2.

The results of comparing Adams and Konigsberg's visual pair-matching study [5], Garrido-Varas and colleagues' geometric morphometric pair-matching study [4], and Byrd and Adams' study on osteometric comparison [6] to the manual and automated MVC methods are found in Table 4. It should be noted that all three studies analyzed different bones and that in the case of Byrd and Adams' study [6], the authors were attempting to associate different bones, such as a humerus to a radius, not just pair-match the same bone. Similarly, as Garrido-Varas and colleague's study cannot detect negatives (i.e., non-pair matches), the specificity cannot be calculated and is effectively $0 \%$ [4].

\section{Discussion}

Ideally, especially in regards to courtroom admissibility and returning more remains to their communities, new tools for distinguishing commingled remain should be highly accurate with known error rates, as determined by statistical certainty. In this study, a new method utilizing digital three-dimensional models of bone was tested to assess its impact on pairmatching skeletal elements accurately. The MVC method

Table 3 Sensitivity and specificity results for the automated mesh-to-mesh value comparison method

\begin{tabular}{ll}
\hline True positives & 42 \\
False negatives & 0 \\
False positives & 0 \\
True negatives & 3 \\
Sensitivity & $100 \%$ \\
Specificity & $100 \%$ \\
\hline
\end{tabular}

\begin{tabular}{ll}
\hline True positives & 38 \\
False negatives & 2 \\
False positives & 2 \\
True negatives & 3 \\
Sensitivity & $95 \%$ \\
Specificity & $60 \%$ \\
\hline
\end{tabular}


Table 4 Sensitivity and specificity results comparing all pair-matching methods

\begin{tabular}{llll}
\hline Method & Authors & Sensitivity & Specificity \\
\hline Visual pair-matching $^{\mathrm{a}}$ & Adams and Konigsberg [7] & $91 \%$ & $100 \%$ \\
Osteometric comparison $^{\mathrm{a}}$ & Byrd and Adams [8] & $42 \%$ & $96 \%$ \\
Geometric morphometrics & Garrido-Varas et al. [6] & $100 \%$ & Not possible \\
Manual MVC & This study & $100 \%$ & $100 \%$ \\
Automated MVC & This study & $95 \%$ & $60 \%$ \\
\hline
\end{tabular}

${ }^{\text {a }}$ Calculations can be found in Online Resources 3 and 4 was invented as a simple attempt to compare the entire surface of two three-dimensional objects and obtain a single value which accurately expresses the similarities between the homologous points of three-dimensional geometry represented. Utilizing the entire geometry of a bone for comparison was expected to improve the results of individualization over the existing methods which use only two-dimensional or partial three-dimensional measurements. Two different versions of the method, one manual and the other automated, were assessed using sensitivity and specificity calculations.

The results obtained from this study have significant implications in light of the demand in forensic anthropology for methods that are more objective and reliable, with known error rates [12]. Foremost, this study uses calculations of specificity and sensitivity to objectively assess the two different versions of the MVC method, in turn creating known error rates for each. Moreover, the manual MVC method seems to outperform the established techniques of visual pair-matching, osteometric comparison, and geometric morphometrics in terms of reliability of pair-matching, with the added benefit of actually having known error rates, unlike visual pairmatching. As the MVC method does not require biological landmarking, it also reduces error across different practitioners. Additionally, another advantage of the method is that it is sex-, population-, and chronology-independent. This drastically simplifies the work of forensic experts when dealing with unclear sets of commingled remains, which can potentially be of different populations or from different time periods. Finally, if the MVC pair-matching method could be applied to mass disasters, it could significantly reduce the cost and burden of DNA testing every bone or bone fragment.
As for a hypothesis regarding why the automated version of MVC is less accurate than the manual version, there are several possible factors. First, though the Flexscan 3D software is based on ICP, from brief experimentation with the software, it also uses additional algorithms to prioritize which surfaces are matched together. Given that the automated MVC method failed to correctly match complete specimens of two different scan types and groups (G1 and G2), the lack of these unknown additional algorithms seems plausible. These algorithms could simply be those for prioritization as mentioned before, or could be other, unknown ones. As the software and its algorithms are proprietary, the exact algorithms may never be known. Second, though different settings for Viewbox, such as point-to-point comparison versus point-to-plane comparison and all of the different iterations of rough and fine alignment, have been tested, other settings, such as different percentages of overlap for cases of fragmentation [26], still need to be investigated. Thus far, the results do not seem to be overly sensitive to the settings, beyond the expected drastic decrease in accuracy of 1 versus $100 \%$ point sampling during the fine alignment stage, but this may change as all of the possibilities of the software are fully explored.

It must also be noted that though the MVC method was tested using two commercial software programs, there is the possibility that the method works just as well on other, free software programs. LMI Technologies, for example, have just released K-Scan, a free program which seems to be identical to Flexscan 3D. Similarly, other common modeling programs such as Mesh Lab or CloudCompare may also work.

Most of the bones tested in this study were complete and in good taphonomic condition, with no visible markers of

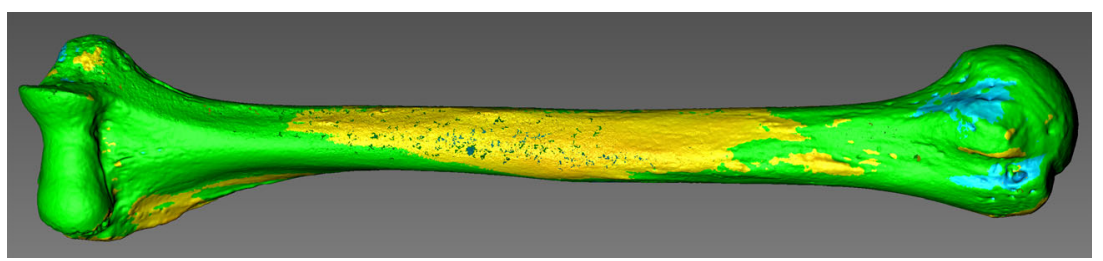

Fig. 2 Deviation analysis feature. Example of the deviation analysis feature, where colors indicate regions of size differences. The green indicates the areas where the two humeri being compared differ by less than $0.645 \mathrm{~mm}$, the yellow where the comparison humerus is $0.654 \mathrm{~mm}$ bigger than the reference humerus, and the blue where the comparison humerus is $0.654 \mathrm{~mm}$ smaller than the reference humerus. Notice how these areas of difference correspond to major muscle attachment features, such as the deltoid tuberosity. Image Credit: Mara Karell (color figure online) 
pathology. This means that the extent and range of the effects of taphonomy, pathology, and fragmentation must be tested for how they impact both the manual and automated MVC method in the future, before it can be applied in the field.

Additionally, scan quality is of the utmost importance when using a method which relies on the accuracy of said scans. Any issues which change the surface quality of a scan, such as improper hole filling from 3D surface scans or CT scan slices that are too thick, will negatively affect the comparison process. Similarly, in our brief experimentation so far, it appears that taphonomy and fragmentation can affect scan quality and thus comparison. Therefore, more research into the extent of these effects must be done before guidelines for scan quality can to be set in order to ensure consistency of results.

Furthermore, the deviation analysis feature in the Flexscan3D software could be explored to help quantify and catalogue different types and prevalence rates of bilateral asymmetry in addition to the MVC method. Once a meshto-mesh value has been created, the deviation analysis feature color-codes the regions of distance between two mesh (such as all of the locations where mesh $\mathrm{A}$ is $0.6 \mathrm{~mm}$ larger than mesh $\mathrm{B}$ are blue, etc.) and allows one to visually inspect similarity. Previous studies on the feature have included measuring the erosion of dinosaur footprints [27]. See Fig. 2 for an example of the deviation analysis feature.

Digital three-dimensional models of bone are permanent, portable, and allow for remote analysis of remains. Though three-dimensional modeling technologies can be expensive, there are a variety of options that range in price, meaning that portable three-dimensional scanners are potentially a field usable tool [20]. As technology progresses and hardware becomes less expensive, it is likely that digital threedimensional models of bones and techniques that utilize these models will be used more and more routinely. Finally, although the MVC method was developed to pair-match human skeletal elements, it can potentially be used as a means of comparison of any two objects with identical or symmetrical components.

\section{Conclusion}

This study tested two different versions of the novel mesh-tomesh value comparison method, one manual and the other automated, for accuracy of pair-matching humeri. Both versions were assessed using sensitivity and specificity calculations. The most effective method was the manual MVC method, utilizing the LMI Technologies Flexscan3D software, which had a sensitivity of $100 \%$ and a specificity of $100 \%$. The automated MVC method, utilizing the Viewbox software, had a sensitivity of $95 \%$ and a specificity of $60 \%$. These values place the manual version of the MVC method among the most accurate methods available for pair-matching skeletal elements. There is further research to be done to improve both versions of the method including testing the effects of taphonomy, pathology, and fragmentation on the process, as well as expanding the method to other paired bones, reducing the overall cost, and fine-tuning the automated comparison algorithms. This study has demonstrated, however, that the meshto-mesh value comparison method is a valuable additional tool for distinguishing commingled human remains.

Acknowledgments The authors would like to thank Ángeles Martín Parrilla and Rosa Gurrea Barricarte for allowing us access to the Ibizan collection, and the Ibiza City Hall for providing the facilities for the osteological study of the remains. We are extremely grateful to Dr. Enrique Lopez for facilitating the use of the CT scanner at the Can Misses Hospital in Ibiza and to Dr. Neil Roberts for scanning the Scottish material at the Clinical Research and Imaging Centre (CRIC), Queens Medical Research Institute (QMRI) in Edinburgh. We would also like to thank Julieta Gómez García-Donas for her translation skills. Finally, we wish to thank all of the reviewers for their invaluable comments and corrections.

\section{Compliance with ethical standards}

Funding This study was partially funded by the Challenge Investment Fund, a part of the University of Edinburgh.

Conflict of interest Author DJH owns stock in dHAL Software, the company that markets Viewbox 4.

Open Access This article is distributed under the terms of the Creative Commons Attribution 4.0 International License (http:// creativecommons.org/licenses/by/4.0/), which permits unrestricted use, distribution, and reproduction in any medium, provided you give appropriate credit to the original author(s) and the source, provide a link to the Creative Commons license, and indicate if changes were made.

\section{References}

1. Adams BJ, Byrd JE (2006) Resolution of small-scale commingling: a case report from the Vietnam War. Forensic Sci Int 156(1):63-69

2. Byrd JE (2008) Models and Methods for Osteometric Sorting. In: Adams BJ, Byrd JE (eds) Recovery, Analysis, and Identification of Commingled Human Remains. Humana Press. pp 199-220. doi:10. 1007/978-1-59745-316-5 10

3. Steadman DW, Adams BJ, Konigsberg LW (2006) Statistical basis for positive identification in forensic anthropology. Am J Phys Anthropol 131(1):15-26

4. Garrido-Varas C, Rathnasinghe R, Thompson T, Savriama Y (2014) A New Method to Pair-match Metacarpals Using Bilateral Asymmetry and Shape Analysis. J Forensic Sci. doi:10.1111/15564029.12577

5. Adams BJ, Konigsberg LW (2004) Estimation of the most likely number of individuals from commingled human skeletal remains. Am J Phys Anthropol 125(2):138-151

6. Byrd JE, Adams BJ (2003) Osteometric sorting of commingled human remains. J Forensic Sci 48(4):717-724

7. Vickers S, Lubinski PM, Henebry DeLeon L, Bowen JT Jr (2014) Proposed Method for Predicting Pair Matching of Skeletal 
Elements Allows Too Many False Rejections. J Forensic Sci. doi: 10.1111/1556-4029.12545

8. Thomas RM, Ubelaker DH, Byrd JE (2013) Tables for the Metric Evaluation of Pair-Matching of Human Skeletal Elements. J Forensic Sci 58(4):952-956. doi:10.1111/1556-4029.12133

9. Auerbach BM, Ruff CB (2006) Limb bone bilateral asymmetry: variability and commonality among modern humans. J Hum Evol 50(2):203-218

10. Steele J, Mays S (1995) Handedness and directional asymmetry in the long bones of the human upper limb. Int J Osteoarchaeol 5(1): 39-49. doi:10.1002/oa.1390050105

11. von Cramon-Taubadel N, Frazier BC, Lahr MM (2007) The problem of assessing landmark error in geometric morphometrics: theory, methods, and modifications. Am J Phys Anthropol 134(1):24 35. doi:10.1002/ajpa.20616

12. Christensen AM, Crowder CM (2009) Evidentiary standards for forensic anthropology. J Forensic Sci 54(6):1211-1216. doi:10. 1111/j.1556-4029.2009.01176.x

13. Rosário Junior AFD, Souza PHC, Coudyzer W, Thevissen P, Willems G, Jacobs R (2012) Virtual autopsy in forensic sciences and its applications in the forensic odontology. Rev Odonto Ciênc 27:5-9

14. O’Donnell C, Iino M, Mansharan K, Leditscke J, Woodford N (2011) Contribution of postmortem multidetector CT scanning to identification of the deceased in a mass disaster: experience gained from the 2009 Victorian bushfires. Forensic Sci Int 205(1-3):1528. doi:10.1016/j.forsciint.2010.05.026

15. Osipov B, Harvati K, Nathena D, Spanakis K, Karantanas A, Kranioti EF (2013) Sexual dimorphism of the bony labyrinth: a new age-independent method. Am J Phys Anthropol 151(2):290301

16. PJ B, ND M (1992) A method for registration of 3-D shapes. IEEE Trans Pattern Anal Mach Intell 14(2):239-256. doi:10.1109/34. 121791
17. Cone EJ, Dickerson S, Paul BD, Mitchell JM (1992) Forensic drug testing for opiates. IV. Analytical sensitivity, specificity, and accuracy of commercial urine opiate immunoassays. J Anal Toxicol 16(2):72-78

18. Casey DG, Price J (2009) The sensitivity and specificity of the

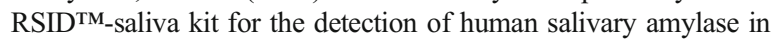
the Forensic Science Laboratory, Dublin, Ireland. Forensic Sci Int 194(1):67-71. doi:10.1016/j.forsciint.2009.10.009

19. Byers SN (2000) Testing type II error rates in biological anthropology. Am J Phys Anthropol 111(2):283-289

20. Friess M (2012) Scratching the surface? The use of surface scanning in physical and paleoanthropology. J Anthropol Sci 90:7-31

21. Hall D, Cachart R (2005) Ballumbie Church (Murroes parish), long cist cemetery; medieval church and graveyard. Discov Excavation Scotl 6:21

22. St Andrews Library: Archaeological Excavation - Data Structure Report (2003). Rathmell Archaeology Ltd, Dundee, Scotland

23. Guerra R, Martín Á (1999) Excavaciones arqueológicas en la Catedral de Eivissa. Eivissa (Ibiza)

24. Spoor CF, Zonneveld FW, Macho GA (1993) Linear measurements of cortical bone and dental enamel by computed tomography: applications and problems. Am J Phys Anthropol 91(4):469-484

25. Loong T-W (2003) Understanding sensitivity and specificity with the right side of the brain. 327:716. doi:10.1136/bmj.327.7417.716

26. Chetverikov D, Svirko D, Stepanov D, Krsek P The Trimmed Iterative Closest Point Algorithm. In: Proceedings of 16th International Conference on Pattern Recognition, 2002. pp 545548. doi:10.1109/ICPR.2002.1047997

27. Adams TL, Strganac C, Polcyn MJ, Jacobs L (2010) High resolution three-dimensional laser-scanning of the type specimen of Eubrontes (?) glenrosensis Shuler, 1935, from the Comanchean (Lower Cretaceous) of Texas: implications for digital archiving and preservation. Palaeontol Electron 13(3):1T-11T 